\title{
Automatic Contrast Phase Estimation in CT Volumes
}

\author{
Michal Sofka ${ }^{1}$, Dijia $\mathrm{Wu}^{1}$, Michael Sühling ${ }^{1}$, David Liu ${ }^{1}$, Christian Tietjen ${ }^{2}$, \\ Grzegorz Soza ${ }^{2}$, and S. Kevin Zhou ${ }^{1}$
}

1 Image Analytics and Informatics, Siemens Corporate Research, Princeton, NJ, USA

2 Computed Tomography, Siemens Healthcare, Forchheim, Germany

\begin{abstract}
We propose an automatic algorithm for phase labeling that relies on the intensity changes in anatomical regions due to the contrast agent propagation. The regions (specified by aorta, vena cava, liver, and kidneys) are first detected by a robust learning-based discriminative algorithm. The intensities inside each region are then used in multi-class LogitBoost classifiers to independently estimate the contrast phase. Each classifier forms a node in a decision tree which is used to obtain the final phase label. Combining independent classification from multiple regions in a tree has the advantage when one of the region detectors fail or when the phase training example database is imbalanced. We show on a dataset of 1016 volumes that the system correctly classifies native phase in $96.2 \%$ of the cases, hepatic dominant phase (92.2\%), hepatic venous phase $(96.7 \%)$, and equilibrium phase $(86.4 \%)$ in 7 seconds on average.
\end{abstract}

\section{Introduction}

Computed Tomography (CT) remains the most common modality used in the imaging of the liver and for the diagnosis of focal liver lesions. Multiphase study obtained by multidetector-row CT (MDCT) during defined circulatory phases best outlines the vasculature and improves detection and characterization of parenchyma lesions [10]. Automatic algorithms for lesion classification, segmentation, and serial comparison require the knowledge of the contrast phase to get the most accurate results (Figure 1). Currently, the phase information either needs to be entered manually or extracted from Dicom tags. Unfortunately, these entries are often incorrect or missing (15-20\% of the cases in our experience, see Section (4), and their format varies across hospitals and clinicians.

In this paper, we present an algorithm for automatic contrast phase classification based on the image intensity in local regions. Since several organs and anatomical structures are enhanced differently
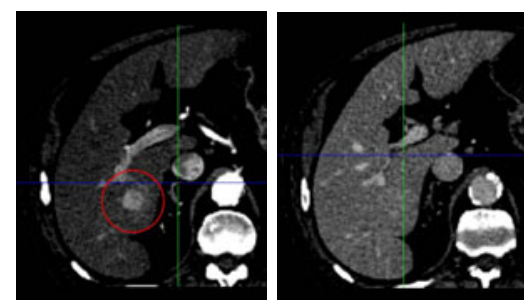

Fig. 1. Contrast phase estimation is important for automatic liver lesion detection and segmentation. The hyperdense liver lesion is clear in the arterial phase (left), but almost invisible in the venous phase (right). 
during a specific contrast phase, the algorithm exploits these different levels of enhancement to automatically determine the phase label. Our approach starts by a robust learning-based detector of anatomical structures. The local regions surrounding the structures are then used to train a classifier identifying the following contrast phases: native (NP), hepatic arterial dominant (HADP), hepatic venous (HVP), and equilibrium (EP). The phases are explained in detail in Section 2

The proposed algorithm uses only the image content to automatically detect the contrast phase without relying on the Dicom tags which often do not contain any label information. This way, the phase labeling is standardized rather than subjective and it can be used in the automatic contrast-specific scan analysis such as lesion detection, segmentation, and comparison in the follow-up studies [26]. Since these algorithms might be inaccurate or even fail when the contrast information is incorrect, the requirements on the phase detection accuracy are high. Moreover, since the contrast estimation is adding computation to the overall scan analysis, the algorithm must be efficient.

The robustness of our algorithm comes from the joint analysis of several local image regions surrounding anatomical structures. Discriminative learning-based anatomy detectors (PBT 9]) are trained using a large annotated database of images. The anatomical structures that we use are the liver, aorta, vena cava at the level of renal artery bifurcation, and kidney center (see Figure 4 for examples). The learning algorithm solves the inherent ambiguity of the anatomy locations (e.g. along the vessel or center of the liver) and approaches performance of the human annotations. Multiple image regions used in the phase classification increases robustness where the phase cannot be determined using one region alone and when one of the anatomy detectors fail.

The proposed phase estimation technique is novel in three aspects. First, it presents a way to effectively combine evidence from multiple image regions using confidence of anatomical detectors. Second, the phase classification procedure is based on a decision tree, where each node is a multi-class LogitBoost classifier. Third, the organization of the tree allows to leverage prior knowledge such as the effectiveness of each landmark to classify particular phase or to adjust the tree topology based on the amount of training labels for each phase. We will show on a database of CT scans that the technique can accurately classify the phase in $93 \%$ of the cases.

\section{Contrast Enhancement in CT}

In the first breath-hold of approximately 24 seconds, the arterial dominant phase (HADP) acquires images with arterial and arterioportal enhancement [5]. In the second breath-hold of about 10 seconds, portal enhancement is scanned beginning 60 seconds after the injection of contrast medium (hepatic venous phase, HVP). During this phase, maximum enhancement of the liver parenchyma and strong enhancement of renal cortex and medulla is achieved. Finally, a 3-min delayed scan (equilibrium phase, EP) is acquired. The renal calices and pelvis 
start to fill with excreted contrast after approximately 120 seconds [4]. Native phase (NP) scan is obtained without contrast injection. Specific enhancement of organs in each of the phases makes it possible to estimate the contrast phase based on the organ scans. Often, only one phase is acquired (see Section 4).

In the clinical routine, contrast phase information is usually added manually to the series description or image comments Dicom fields. Unfortunately, this information is not structured or standardized. The new Dicom objects aimed to capture timing and phase of enhancement [1] are not widely adopted and there already exists a vast amount of data with unstructured tags. The acquisition timing in the image meta data (if available) could be used to extract time delay between multiple scans. However, the delay after the start of contrast injection could not be obtained since the power injector for the agent is not coupled with the scanner. Moreover, all phases are not always scanned to reduce the amount of radiation to the patient. In our approach, we do not rely on the meta data but rather use the image regions surrounding liver, aorta, vena cava (at the renal vein branching), and kidneys to automatically estimate the contrast phase.

To illustrate the image intensity changes in various contrast phases, we computed the following statistics. Each anatomical region was used to obtain a histogram of intensities and a statistical value of the histogram was plotted for all volumes (Figure 2). We used the mean value of the histogram for aorta and vena cava and peak value for liver and kidneys (due to intensity inhomogeneities). The figure shows, that the regions are indicative of the contrast phase. However, it is not possible to classify all the phases by one of the regions alone as evidenced by overlaps in the plots.
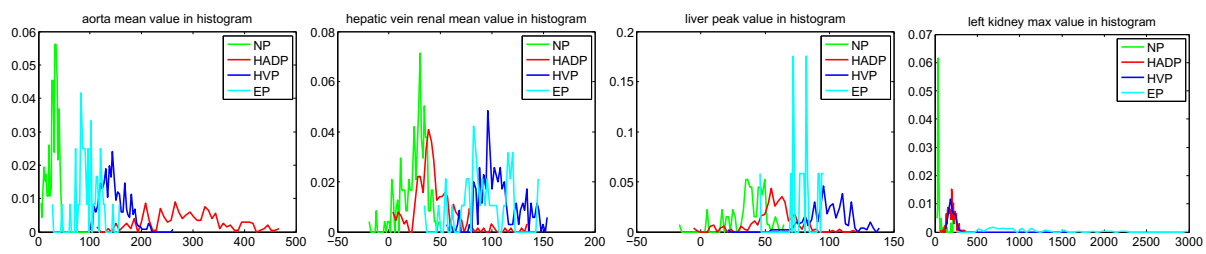

Fig. 2. Intensity statistics computed from 1016 volumes within the image regions defined by aorta, vena cava, liver, and left kidney (right kidney plot is similar). The intensities within the selected anatomical structures are indicative of the contrast phase.

\section{Algorithm}

Our algorithm automatically determines a probabilistic estimate of the image contrast phase. The set of phases $\mathbb{P}$ that we estimate is composed of hepatic dominant phase (HADP), hepatic venous phase (HVP), native phase (NP) (without contrast injection), and equilibrium phase (EP). The algorithm uses a set of observations for contrast phase $r_{i}$ obtained from the neighborhoods $V_{1}, V_{2}, \ldots, V_{t}$ of the image $V$. Each neighborhood $V_{j}$ is specified by the coordinates of a bounding box within a $d$-dimensional image $V, V: R^{d} \rightarrow[0,1]$. The set of observations 
describe the intensity appearance specific for each region and phase. The goal of the phase contrast detection algorithm is to estimate for a given volume $V$ the probability mass function $p\left(r_{i} \mid V\right)$ for each contrast phase $r_{i} \in \mathbb{P}$.

Estimating the phase $r_{i}$ becomes difficult when the number of phases in the set $\mathbb{P}$ is large; it is easier to distinguish between two phases than among four. Furthermore, not all observations are useful for classification of each phase, especially when the observations are the same for two or more phases (this is a case for kidney regions as can be seen from Figure 21). To address this problem, we propose a multi-level algorithm, where the number of phases $\left|\mathbb{P}_{s}\right|$ at each level $s$ is smaller, $\mathbb{P}_{s} \subset \mathbb{P}$, and the set of observations is also smaller. In our experiments, we found that two levels achieve reliable contrast phase estimation (Figure 3). In this case, we can write

$$
p\left(r_{i} \mid V\right)=\sum_{k} p\left(r_{i} \mid V, q_{k}\right) p\left(q_{k} \mid V\right)
$$

where $r_{i}$ and $q_{k}$ are contrast phases estimated at level 2 and level 1 , respectively.

We estimate the contrast using neighborhood image regions surrounding liver, aorta, vena cava, and kidneys. Each oriented region $j$ is defined by the parameters $\boldsymbol{\theta}_{j}=\{\mathbf{p}, \mathbf{r}, \mathbf{s}\}$, that specify the position $(\mathbf{p})$, orientation $(\mathbf{r})$, and size (s) of the region. The set of observations inside the image neighborhood $V_{j}$ is taken from the region defined by $j$ and therefore

$$
p\left(q_{k} \mid V\right)=\sum_{j} p\left(q_{k} \mid V, j\right) p(j)
$$

Prior distribution $p(j)$ of regions surrounding anatomical structures is uniform. The term $p\left(q_{k} \mid V, j\right)$ specifies distribution of contrast phase $q_{k}$ for region $j$. Furthermore, since the pose of the anatomy $j$ is defined by parameters $\boldsymbol{\theta}_{j}$

$$
\begin{aligned}
p\left(q_{k} \mid V, j\right) & =\int p\left(q_{k}, \boldsymbol{\theta}_{j} \mid V\right) d \boldsymbol{\theta}_{j} \\
& =\int p\left(\boldsymbol{\theta}_{j} \mid V\right) p\left(q_{k} \mid \boldsymbol{\theta}_{j}, V\right) d \boldsymbol{\theta}_{j} .
\end{aligned}
$$
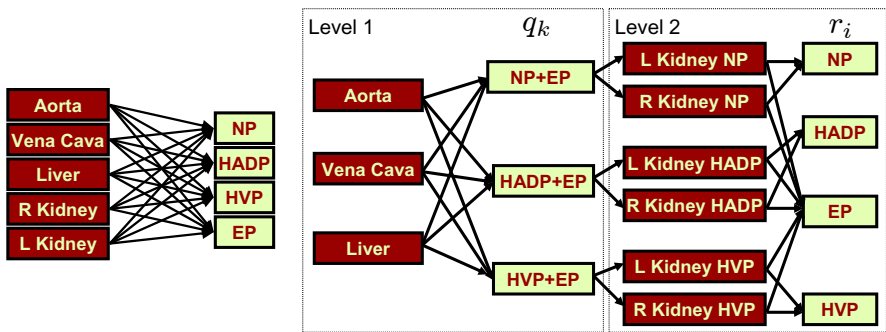

Fig. 3. Multiple level algorithm (right) has advantages compared to a single level algorithm (left) when the phase training data is imbalanced and effectively exploits discriminative power of each landmark. In our case, kidneys are useful for classifying EP phase but not the other phases (as seen in Figure 2). 
In practice, we replace the integration over the parameters by the best instance

$$
p\left(q_{k} \mid V, j\right) \approx p\left(\hat{\boldsymbol{\theta}}_{j} \mid V\right) p\left(q_{k} \mid \hat{\boldsymbol{\theta}}_{j}, V\right) .
$$

The set of the best instance parameters $\hat{\boldsymbol{\theta}}_{j}$ for each anatomical region $j$ is estimated using a volumetric context surrounding the anatomy position:

$$
\hat{\boldsymbol{\theta}}_{j}=\arg \max _{\boldsymbol{\theta}_{j}} \mathrm{P}\left(\boldsymbol{\theta}_{j} \mid V\right)
$$

where $\mathrm{P}\left(\boldsymbol{\theta}_{j} \mid V\right)$ is the probability of the parameters given the image volume. Let us now define a random variable $y \in\{-1,+1\}$, where $y=+1$ indicates the presence and $y=-1$ absence of the anatomy. We train a Probabilistic Boosting Tree classifier (PBT) [9] with nodes composed of AdaBoost classifiers trained to select Haar and steerable features [8] that best discriminate between positive and negative examples of the anatomy. We can then evaluate the probability of an anatomy being detected as $\mathrm{P}\left(y=+1 \mid \boldsymbol{\theta}_{j}, V\right)$. A natural choice for Eq. 6 is to use the $\mathrm{P}\left(y=+1 \mid \boldsymbol{\theta}_{j}, V\right)$,

$$
\hat{\boldsymbol{\theta}}_{j}=\arg \max _{\boldsymbol{\theta}_{j}} \mathrm{P}\left(y=+1 \mid \boldsymbol{\theta}_{j}, V\right) .
$$

This way, we sucessfuly convert the above problem to a detection problem. The derivation for $p\left(q_{k} \mid V\right)$ applies to all levels of the algorithm.

We use a Multi-class LogitBoost (MLBoost) [3] classifier and a Haar feature selection from AdaBoost [3] to estimate the contrast phase distribution $p\left(q_{k} \mid \hat{\boldsymbol{\theta}}_{j}, V\right)$ in Eq. 5 . The MLBoost is a generalization of a two-class AdaBoost, interpreted using the forward additive logistic regression. The LogitBoost algorithm uses quasi-Newton steps 7 for fitting an additive symmetric logistic model by maximum-likelihood. At each iteration, the algorithm increases the classification accuracy on the training data by adding a new function $f_{k m}\left(\hat{\boldsymbol{\theta}}_{j}, V\right)$ to the response function $F_{k}\left(\hat{\boldsymbol{\theta}}_{j}, V\right)$. The output of the training is a set of response functions, one for each phase $q_{k}$

$$
F_{k}\left(\hat{\boldsymbol{\theta}}_{j}, V\right)=\sum_{m} f_{k m}\left(\hat{\boldsymbol{\theta}}_{j}, V\right) .
$$

The posterior phase probability $p\left(q_{k} \mid \hat{\boldsymbol{\theta}}_{j}, V\right)$ is then given by

$$
p\left(q_{k} \mid \hat{\boldsymbol{\theta}}_{j}, V\right)=\frac{\exp \left(F_{k}\left(\hat{\boldsymbol{\theta}}_{j}, V\right)\right)}{\sum_{i} \exp \left(F_{i}\left(\hat{\boldsymbol{\theta}}_{j}, V\right)\right)} .
$$

The functions $\left\{f_{k m}\right\}$ are assumed piecewise constant functions of responses computed using a set of weak classifiers selected incrementally during boosting [3].

\section{Experiments}

Our experiments evaluate the accuracy of individual region detectors and final classification performance. 
The data set consists of $1016 \mathrm{CT}$ scans with sizes ranging from $512 \times 512 \times 38$ to $512 \times 512 \times 512$ voxels and resolutions ranging from $0.52 \times 0.52 \times 0.5$ to $1.27 \times 1.27 \times 5.0 \mathrm{~mm}$ resampled to a $3 \mathrm{~mm}$ isotropic resolution (sufficient for phase estimation application). The images were annotated by an expert, resulting in the landmark and phase annotation counts summarized in Table 1. The phase label in the Dicom tags is incorrect in $6.4 \%$ and missing in $9.1 \%$ of the cases (total of $15.5 \%$ ) 1 . Total of $61.8 \%, 29.3 \%$, and $8.9 \%$ studies have scans from one, two, and three phases, respectively. No study contains scans from all four phases.

Table 1. Number of annotated volumes organized by each landmark and phase. The least number of volumes was obtained during the EP phase since it is not scanned as often as the other phases.

\begin{tabular}{c|cccccc} 
& \multicolumn{1}{|c|}{ Native } & HAP & PVIP & HVP & EP & Total \\
\hline Liver & 81 & 152 & 128 & 209 & 17 & 587 \\
Aorta & 239 & 152 & 125 & 349 & 45 & 910 \\
Vena Cava & 174 & 135 & 104 & 177 & 42 & 632 \\
Left Kidney & 159 & 113 & 120 & 304 & 50 & 746 \\
Right Kidney & 146 & 158 & 122 & 314 & 55 & 795 \\
\hline By Phase & 242 & 174 & 141 & 378 & 81 & 1016
\end{tabular}

In the first experiment, we assess the performance of the anatomical structure detectors. The data set with structure annotations was separated randomly into two disjoint sets, one for training (70\% of volumes) and one for testing $(30 \%)$. Each detector was trained using the training data set of all available contrast phases. The detectors were evaluated on the testing data set and the results compared against the annotations. The training errors of each landmark are shown in Table 2. The detection errors are low overall. Occasionally, a detector can have a larger error but this still does not mean the phase classification will be incorrect since we are using evidence from multiple anatomical regions (Eq. 55).

Table 2. Accuracy of the region detectors. The errors for vena cava are larger than aorta due to similar intensity as the liver parenchyma in NP. The vessel position and size errors along the vessel and angle rotations around the vessel are not considered due to ambiguity. The errors for kidneys and liver are higher due to their larger sizes.

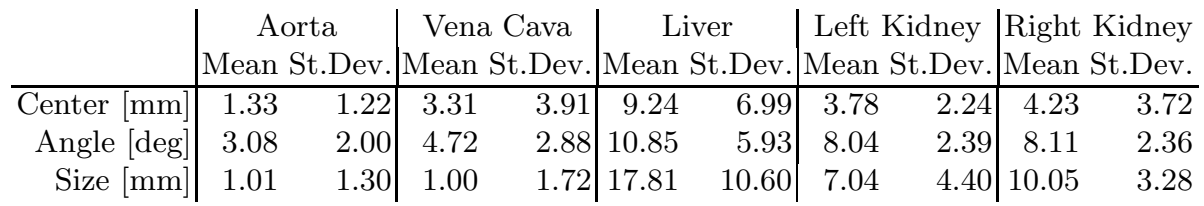

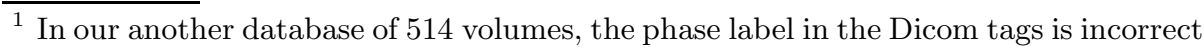
in $5.8 \%$ and missing in $14.7 \%$ of the cases (total of $20.5 \%$ ). We manually removed the language, formatting, and abbreviation ambiguities of the Dicom entries.
} 

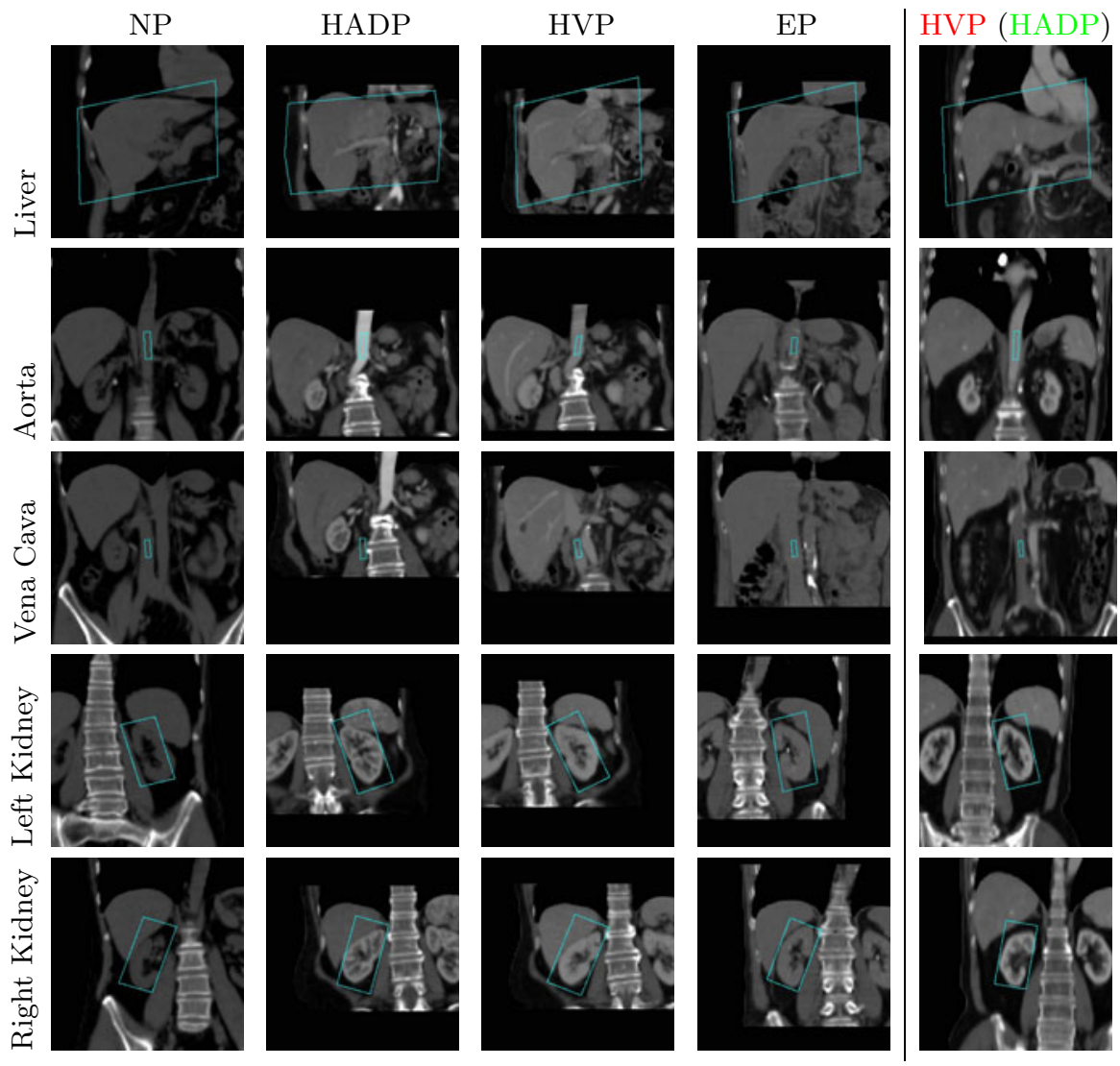

Fig. 4. Detected anatomical structures (rows) used in contrast phase estimation (cols 1-4). Anatomy enhancement specific to each phase can be clearly seen. Incorrectly classified HADP as a HVP phase for a scan in phase transition (5th col.). The lower contrast of aorta and the beginning of liver parenchyma, renal cortex and renal medulla enhancement are characteristic for a HVP phase (compare to 2nd and 3rd col.).

Finally, we present the results of the contrast phase estimation. We trained Multi-class LogitBoost 3] phase classifiers using the annotated anatomical regions. The final phase classification performance after 4 -fold cross validation is summarized in a confusion Table 3. The classification accuracy is high for NP, HADP, and HVP phases, ranging between $92.2 \%$ and $96.7 \%$. For the EP phase, the performance is lower due to the low number of training examples. The EP phase is most often confused with HVP phase which is caused by different parts of kidney being enhanced during these phases (cortex and medulla in HVP and calices and pelvis in EP). It might be possible to improve the result by segmenting these regions to separate them for phase classifier training. The overall phase classification speed is 7 seconds on average. Example qualitative anatomical structure detection result and phase classification are shown in Figure 4 . 
We also compared the two-level algorithm to a single level system where each anatomical region is used to estimate all phases (Figure 3). This system has advantage compared to estimating the phase from all regions jointly since the region detection might fail. However, due to imbalanced training set (Table 1) and poor discrimination of phases in kidneys (Figure 2), the phase was correctly classified only in $85 \%$ cases with correct EP classification only in $29 \%$ cases.
Table 3. Confusion table showing percentages of correctly and incorrectly identified phases when compared to the ground truth (GT) labeling.

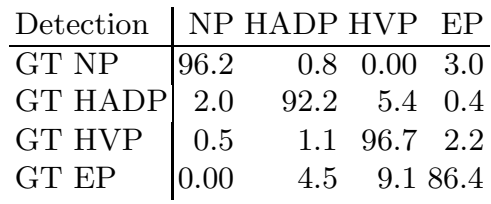

\section{Conclusion}

We presented an automatic phase classification algorithm in CT volumes. Our approach starts by a discriminative learning-based detector of anatomical structures. The regions surrounding the structures are used in Multi-class LogitBoost classifiers to accurately characterize the contrast phase. The system robustly classifies native phase (correct classification in $96.2 \%$ of the cases), hepatic dominant phase (92.2\% correct), hepatic venous phase $(96.7 \%$ correct), and equilibrium phase $(86.4 \%$ correct). The overall speed is 7 seconds on average.

In future, we plan to exploit the time relationship between phases. This will help when there are scans from multiple phases available. We will also investigate the possibility of using pairs of anatomical regions during classification. This will limit the influence of intensity differences across regions for a particular phase as the classifier would focus on relative intensity values computed within the pair of the regions rather than on absolute intensity values in each region.

\section{References}

1. Clunie, D.: Impact of new DICOM objects on handling large data sets. In: University of Rochester PACS 2006 Conference, San Antonio, Texas (March 2006)

2. Deng, X., Du, G.: Editorial: Liver tumor segmentation. In: 3D Segmentation in the Clinic: A Grand Challenge II, MICCAI Workshop, New York, NY (2008)

3. Friedman, J., Hastie, T., Tibshirani, R.: Additive logistic regression: A statistical view of boosting. Annals of Statistics 28(4), 337-407 (2000)

4. Graser, A., Staehler, M., Reiser, M.F., Mueller-Lisse, U.G.: Low-dose multiphasic protocols offer the best option for cancer detection, classification, and staging. Diagnostic Imaging (April 14, 2006)

5. Laghi, A., Sansoni, I., Celestre, M., Paolantonio, P., Passariello, R.: Computed tomography. In: Lencioni, R., Cioni, D., Bartolozzi, C. (eds.) Focal Liver Lesions: Detection, Characterization, Ablation. Springer, Heidelberg (2005)

6. Meyer, C., Park, H., Balter, J., Bland, P.: Method for quantifying volumetric lesion change in interval liver CT examinations. IEEE TMI 22(6), 776-781 (2003)

7. Nocedal, J., Wright, S.J.: Numerical Optimization. Springer, Heidelberg (2000) 
8. Sofka, M., Zhang, J., Zhou, S., Comaniciu, D.: Multiple object detection by sequential Monte Carlo and hierarchical detection network. In: CVPR (2010)

9. Tu, Z.: Probabilistic boosting-tree: Learning discriminative models for classification, recognition, and clustering. In: CVPR, vol. 2, pp. 1589-1596 (2005)

10. Valette, P.J., Pilleul, F., Crombé-Ternamian, A.: Imaging benign and metastatic liver tumors with MDCT. In: Marchal, G., Vogl, T., Heiken, J.P. (eds.) Multidetector-Row Computed Tomography: Scanning and Contrast Protocols. Springer, Heidelberg (2005) 